January 2008

\title{
Management of the neck in differentiated thyroid cancer
}

\author{
David M. Cognetti \\ University of Pittsburgh Medical Center \\ Edmund A. Pribitkin \\ Thomas Jefferson University \\ William M. Keane \\ Thomas Jefferson University
}

Follow this and additional works at: https://jdc.jefferson.edu/otofp

Part of the Otolaryngology Commons

\section{Let us know how access to this document benefits you}

\author{
Recommended Citation \\ Cognetti, David M.; Pribitkin, Edmund A.; and Keane, William M., "Management of the neck in \\ differentiated thyroid cancer" (2008). Department of Otolaryngology - Head and Neck Surgery \\ Faculty Papers. Paper 3. \\ https://jdc.jefferson.edu/otofp/3
}

This Article is brought to you for free and open access by the Jefferson Digital Commons. The Jefferson Digital Commons is a service of Thomas Jefferson University's Center for Teaching and Learning (CTL). The Commons is a showcase for Jefferson books and journals, peer-reviewed scholarly publications, unique historical collections from the University archives, and teaching tools. The Jefferson Digital Commons allows researchers and interested readers anywhere in the world to learn about and keep up to date with Jefferson scholarship. This article has been accepted for inclusion in Department of Otolaryngology - Head and Neck Surgery Faculty Papers by an authorized administrator of the Jefferson Digital Commons. For more information, please contact: JeffersonDigitalCommons@jefferson.edu. 
Management of the neck in differentiated thyroid cancer

David M Cognetti, MD ${ }^{\mathrm{a}}$, Edmund A. Pribitkin, $\mathrm{MD}^{\mathrm{b}}$, and William M. Keane, $\mathrm{MD}^{\mathrm{c}}$

${ }^{\mathrm{a}}$ Fellow, Head and Neck Surgery, Department of Otolaryngology-Head and Neck Surgery,

University of Pittsburgh Medical Center, Pittsburgh, PA

${ }^{b}$ Professor and Academic Vice-Chairman, Department of Otolaryngology-Head and Neck

Surgery, Thomas Jefferson University, Philadelphia, PA

${ }^{c}$ Professor and Chairman, Department of Otolaryngology-Head and Neck Surgery, Thomas

Jefferson University, Philadelphia, PA

Keyword: differentiated thyroid cancer, thyroid carcinoma, neck dissection, radioactive iodine, management guidelines

${ }^{\mathrm{a}}$ Corresponding author for

proof and reprints:

David M Cognetti, MD

Department of

Otolaryngology-Head

and Neck Surgery

Thomas Jefferson

University

925 Chestnut Street, $6^{\text {th }}$

Floor

Philadelphia, PA 19107

(215) 955-6784

(215) 923-4532 (fax)

david.cognetti@gmail.com
${ }^{\mathrm{b}, \mathrm{c}}$ Coauthors Addresses:

Edmund A Pribitkin, MD

William M. Keane, MD

Department of

Department of

Otolaryngology-Head

Otolaryngology-Head

and Neck Surgery

and Neck Surgery

Thomas Jefferson

Thomas Jefferson

University

University

925 Chestnut Street, $6^{\text {th }}$

925 Chestnut Street, $6^{\text {th }}$

Floor

Floor

Philadelphia, PA 19107

(215) 955-6784

(215) 923-4532 (fax)

Edmund.pribitkin@jefferson.edu
(215) 955-6784

(215) 923-4532 (fax)

Philadelphia, PA 19107

William.keane@jefferson.edu 


\section{Synopsis}

Differentiated thyroid cancer is characterized by an excellent long-term prognosis which unlike other head and neck carcinomas is not definitively influenced by regional lymph node metastasis. The relative rarity of the disease coupled with its tendency for delayed metastatis and its low mortality make a prospective randomized trial comparing treatment outcomes difficult. As a result, the effect of cervical lymph node metastases on survival is unclear making meaningful recommendations for management somewhat subjective and arbitrary. The purpose of this review is to discuss guidelines for the management of the neck in differentiated thyroid cancer while reviewing the available literature.

\section{Introduction}

The American Cancer Society estimates that there will be 33,550 new cases of thyroid cancer for 2007, representing approximately $2 \%$ of all cancers in the United States. ${ }^{1}$ Differentiated thyroid carcinoma (DTC) includes papillary thyroid carcinoma (PTC) and follicular thyroid carcinoma (FTC), which are both derived from thyroid follicular cells. DTC comprises approximately $90 \%$ of all thyroid cancers, and carries an excellent long-term prognosis. ${ }^{2}$ In fact, two large database studies involving 15,698 and 53,856 cases of thyroid carcinoma treated in the United States demonstrated a 10-year survival rate for PTC of $98 \%$ and $93 \%$, respectively, and a 10-year survival rate for FTC

of $92 \%$ and $85 \%$, respectively. ${ }^{3,4}$ Unlike squamous cell carcinoma of the head and neck, where regional metastases have a definite negative prognostic impact, the effect of cervical nodal involvement on survival in DTC has not been clearly demonstrated. The 
high survival rate combined with the unclear impact of regional metastases has resulted in inconsistent approaches to the management of the neck in thyroid cancer over the years. Indeed, the relative rarity of the disease, combined with its favorable prognosis, limits the ability to compare different treatment protocols by prospective randomized clinical trials. ${ }^{3}$ If long-term survival is the standard metric of all treatment options, then the goal of limiting morbidity and recurrence becomes even more important. Therefore, the management of the neck in DTC must take these factors into account.

In 2006, the American Thyroid Association (ATA) Guidelines Taskforce released updated recommendations for the management of DTC, including guidelines for the management of the neck. ${ }^{5}$ The purpose of this review is to expand upon those guidelines while discussing the evidence leading to those recommendations and highlighting special considerations and newer techniques proposed for the management of the neck in thyroid carcinoma. The focus of this review will be on DTC, which comprises the vast majority of thyroid cancers.

\section{Background}

\section{Pattern of nodal metastasis}

Lymph node metastases occur frequently in PTC. A review of 1,038 previously untreated patients with DTC who presented to Memorial Sloan Kettering over a 55-year period showed the incidence of nodal metastasis in PTC to be $56 \% .^{6}$ Several more recent studies have confirmed the incidence of nodal metastasis in PTC to be in the range of 60$65 \% .^{7-10}$ Ito et al ${ }^{11}$ investigated the rate of nodal metastasis from papillary thyroid microcarcinoma (PTMC), defined as tumor size $\leq 10 \mathrm{~mm}$, and still found an incidence 
close to $50 \%$. Qubain et $\mathrm{al}^{12}$ investigated the lymph nodes of 80 patients with DTC that were deemed pathologically N0 by standard hematoxylin and eosin staining. Of the 71 patients with PTC, 54\% had lymph node micrometastases when examined immunohistochemically with cytokeratins (AE1/AE3). Lymph node metastases in FTC occur less frequently. In their 55 -year review, Shaha et al reported $21 \%$ with cervical metastases at presentation. ${ }^{6}$

Several studies have attempted to define the pattern of lymph node metastases for PTC, as has been demonstrated for squamous cell carcinoma of the head and neck. ${ }^{10,13-16}$ Goropoulos et a ${ }^{10}$ studied 39 patients in whom a central neck dissection (CND) was performed. Some of these patients also had a lateral neck dissection (LND) and all patients had post-operative I-131 scans. They discovered that metastasis to the central compartment was common (64\%), as was metastasis to the ipsilateral cervicolateral compartment (51.2\%.) Contralateral cervicolateral metastasis occurred in $33.3 \%$ of the patients and never occurred without associated metastases to the central compartment and the ipsilateral side. None of the patients studied had disease in the lateral compartments while lacking metastasis to the central compartment, leading the authors to suggest that the nodal status of the central compartment is a valuable indicator of lymphatic involvement of the lateral compartments.

Gimm et $\mathrm{al}^{13}$ also demonstrated that the cervicocentral compartment was the most frequent site of metastasis, but differentiated between ipsilateral and contralateral central compartments. Of the 35 patients studied, 24 had metastasis to the ipsilateral cervicocentral compartment, while only 5 had metastases to the contralateral cervicocentral compartment. All five of these patients had T3 or T4 tumors. Nineteen 
patients had metastases to the ipsilateral cervicolateral compartment while no patients had metastases to the contralateral cervicolateral compartment and only 1 patient had a mediastinal metastasis. Contrary to the suggestion of Goropoulos et $\mathrm{al}^{10}$ advocating the central compartment as an indicator of lateral involvement, Gimm at $\mathrm{el}^{13}$ discovered ipsilateral cervicolateral metastases in the absence of central metastases in 5 patients.

At a Japanese institution where neck dissections are performed routinely for all sized PTC, a review of 259 cases of PTMC confirmed the preference of metastases to spread to the central $(64 \%)$ and ipsilateral nodes $(44.5 \% \text {. })^{8}$ They did not clarify whether the status of the central compartment was predictive of the lateral compartment, but they did further divided the central compartment to report the incidence of pretracheal (43.2\%), ipsilateral (36.3\%), and contralateral (18.9\%) positive nodes. Other groups have further characterized the pattern of lymph node metastases from PTC to the lateral nodes. Kupferman et $\mathrm{al}^{14}$ reported the distribution of positive nodes from 44 therapeutic neck dissections, and found levels II (52\%), III (57\%), and IV (41\%) to be most frequently involved. However, the incidence of level V nodes (21\%) was high enough for the authors to advocate a comprehensive (level II through V) neck dissection when lateral metastases are present. Sivanandan and $\mathrm{Soo}^{16}$ analyzed specimens from 80 therapeutic neck dissections and had similar results and conclusions.

The rate and number of nodal metastases in PTC have been shown to be associated with the size of the thyroid tumor..$^{9,15,17}$ Ito et al $^{9}$ studied 759 cases of PTC and showed that the frequency of metastasis to the central compartment was $38.3 \%$ for tumors measuring $1 \mathrm{~cm}$ or smaller, and rose significantly to $79.0 \%$ for tumors larger than $4 \mathrm{~cm}$. Like Gimm ${ }^{13}$, they found some patients $(11.2 \%)$ to be positive for lateral but negative for 
central node metastasis, questioning the reliability of the central compartment as an indicator for lateral involvement.

\section{Effect of nodal metastases on survival}

As demonstrated above, it is clear that lymph node metastasis in PTC is frequent and has a predictable pattern. However, it remains controversial whether the presence of lymph node metastases, either overt or occult, has an impact on prognosis. It is this fact that has prevented adoption of a universal approach to management of the neck in thyroid cancer.

Traditionally, it has been suggested that the presence of regional metastases has no effect on survival. ${ }^{18-24}$ Hughes et al ${ }^{19}$ performed a matched-pair analysis of patients with differentiated thyroid cancer and N0 versus NI neck involvement. There were 100 patients in each group, and they were matched according to age, tumor size, histology, and intrathyroidal extent. All lacked distant metastases. There was no significant difference in survival. Shaha et $\mathrm{al}^{18}$ and McConahey et $\mathrm{al}^{20}$ each presented retrospective series of over 800 patients and also found that the presence of lymph node metastases had no effect on

survival. Scheuman et $\mathrm{al}^{25}$ found that N1 status in PTC did influence survival for patients with T1-T3 status, but not for those with T4 status. A more recent nested case-controlled study suggests that lymph node metastasis does have an adverse effect on prognosis. ${ }^{26}$ Mazzaferri et $\mathrm{al}^{21}$ found that cervical metastases carried a higher cancer mortality rate in patients with FTC, but not in patients with PTC.

Increasing age, male gender, distant metastases, extrathyroidal spread, and large

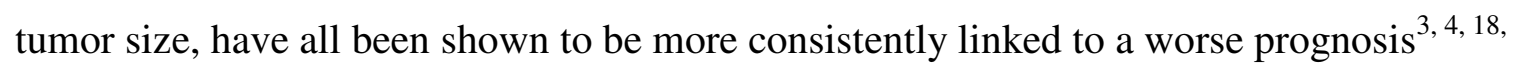


20,21 In fact, when univariate analysis is applied to the presence of regional lymph nodes, some have found it to be a favorable prognostic indicator. ${ }^{19}$ Although this appears to be counterintuitive, the finding can be explained by the positive influence of younger age. PTC in younger patients is often associated with nodal metastases. In this instance, age appears to influence survival more than metastatic disease, and therefore biases the results. A study comparing patients of the same age with and without metastatic disease would more properly address this question. Such a study in older patients has demonstrated an adverse impact of nodal disease on survival. $^{27}$

\section{Effect of nodal metastases on recurrence}

Up to $30 \%$ of patients with DTC develop recurrent metastatic cervical disease with minimal adverse impact on survival..$^{21,23,28}$ Patients with nodal disease at presentation have a higher incidence of recurrent disease in the cervical region. Ito et $\mathrm{al}^{9}$ evaluated 626 patients with papillary carcinoma whose primary tumor was larger than $1 \mathrm{~cm}$ using multivariate analysis. They found that patients with central node metastasis had an independently significant decreased disease-free survival when compared to those without metastasis. The size and number of the metastatic nodes were also demonstrated to impact recurrence. In McConahey's ${ }^{20}$ study of 859 patients over a 24 -year treatment period, the presence of metastatic nodes at the time of initial examination was associated with a 10fold increase in the risk of developing a nodal recurrence. Scheumann et $\mathrm{al}^{25}$ demonstrated that $\mathrm{N} 1$ patients had a significant increase in recurrence when compared to N0 patients with T1-T3 tumors. In Hughes et al's ${ }^{19}$ matched-pair analysis the recurrence rate was higher in $\mathrm{N} 1$ patients $(17 \%)$ than in N0 patients (11\%), but did not reach 
significance. However, when their analysis was limited to patients over the age of 45, the recurrence in $\mathrm{N} 1$ patients was $31 \%$ and was significantly greater than in the N0 patients $(8 \%)$

The effect of metastases, whether occult or overt, on prognosis and recurrence has important implications for the management of the neck. This is further discussed below in the context of indications for neck dissection.

\section{Preoperative}

\section{Fine-Needle Aspiration}

Fine needle aspiration (FNA) is considered the procedure of choice in the evaluation of thyroid nodules according to the ATA Guidelines Taskforce. ${ }^{5}$ The procedure is minimally invasive, accurate, and cost effective. FNA also plays an important role in the workup of a neck mass. Cervical adenopathy can be the presenting finding in patients with DTC, especially young people with PTC. ${ }^{29}$ FNA is advocated in the pre-operative workup of a neck mass to aid in appropriate diagnosis and pre-operative planning. It potentially differentiates metastatic PTC from metastatic squamous cell carcinoma, thus allowing an appropriately directed search and treatment of the respective

primary tumor and neck disease. In younger patients, lymphoma is often suspected and an excisional biopsy of the cervical node may be performed. Cervical metastases are common in younger patients with PTC, however, and FNA could allow for differentiation from lymphoma prior to excisional biopsy, thus preventing a potentially inappropriate procedure. Finally, measurement of thyroglobulin in a $1 \mathrm{ml}$ saline wash-out of the needle used for the biopsy has been shown to be an easy, inexpensive, and reliable method of 
increasing the diagnostic yield of FNA. ${ }^{30,31}$ This is especially true of cystic lymph nodes, which frequently result in nondiagnostic cytology. ${ }^{32}$

\section{Ultrasound, MRI, CT}

Preoperative staging of the neck aids in surgical decision-making for DTC.

Physical examination alone can underestimate the involvement of regional lymph nodes, which is quite frequent in PTC. Ultrasonography is non-invasive, well-tolerated, and improves the identification of cervical adenopathy. Kouvaraki et $\mathrm{al}^{28}$ at the University of Texas M.D. Anderson Cancer Center retrospectively reviewed 212 patients with thyroid carcinoma who underwent preoperative ultrasonography. They reported that ultrasonography detected lymph node or soft tissue metastases in neck compartments believed to be uninvolved by physical exam in $39 \%$ of the patients. Thus, ultrasonography altered the surgical procedure in these patients, potentially minimizing recurrence in the neck.

Although the specificity of ultrasonography for cervical lymph node metastases is very high, its sensitivity has been shown to be as low as $36.7 \%$ for lateral compartments and as low as $10.9 \%$ for the central compartments. ${ }^{33-35}$ Interestingly, Ito et al $^{33}$ examined the usefulness of preoperative ultrasonographic examination of the neck in patients with PTMC and determined that only the lateral compartment nodes that were identified on ultrasound preoperatively had clinical relevance, mitigating the concern for low sensitivity of the technique. They reviewed 590 patients, 67 of which had evidence of metastases on preoperative ultrasound. These 67 all underwent a modified radical neck dissection (MRND) as part of their management. Of the 523 patients without preoperatively 
detectable metastasis, 249 underwent MRND and 274 did not. Despite the fact that more than $40 \%$ of the patients who underwent MRND had pathologic evidence of metastasis, the MRND and observations group did not statistically differ in lymph node recurrence rates $(<1.5 \%$ for both) when followed for a mean of over 4 years.

It should be noted that the sonographic criteria for identifying lymph nodes suspicious for metastases is not limited to lymph node size. Architectural features of the

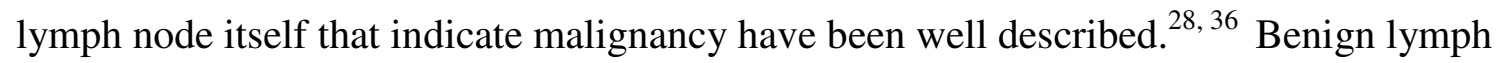
nodes are typically oval and flattened with a smooth cortex and a central fatty hilum. Features that can indicate malignancy include a rounded bulging shape, replaced fatty hilum, eccentric cortical widening, irregular margins, decreased or heterogeneous echogenicity, alteration in intranodal vascularity, cystic areas, and calcifications. These findings can be present even in small nodes and are not appreciated on simple palpation.

One limitation of ultrasonograpy is that it is operator dependant. However, it is less expensive than computed tomography and magnetic resonance imaging. Additionally, the 2006 ATA Guidelines recommend ultrasound of the thyroid for the workup of all patients with one or more suspicious thyroid nodules. ${ }^{5}$ Preoperative neck ultrasound to evaluate the contralateral thyroid lobe as well as cervical lymph nodes is also recommended for all patients undergoing thyroidectomy for malignant cytologic findings on biopsy. An experienced sonographer, with appropriate communication from the referring specialist, can potentially extend a diagnostic thyroid ultrasound to include surveillance of the lateral compartments when suspicion for malignancy is heightened by patient history, by sonographic features of the thyroid nodule, or by preliminary cytologic findings of the thyroid nodule if ultrasound guided FNA is being performed. This would 
minimize the time required and number of interventions needed during the preoperative workup. Although the ATA guidelines suggest that CT and MRI may be useful in some clinical settings (large, rapidly growing, and invasive tumors), they do not recommend the routine preoperative use of these studies. ${ }^{5}$ If computed tomography is utilized, it is important to remember that iodinated contrast dye can interfere with radioactive iodine uptake, and thus should be avoided when performing a neck CT with suspicion of thyroid cancer.

\section{FDG-PET}

Positron emission tomography (PET) has gained popularity in the diagnostic workup and post-treatment surveillance of squamous cell carcinoma of the head and neck. Its role in thyroid carcinoma has not been clearly delineated and the ATA guidelines do not recommend its use pre-operatively. Early studies suggest, however, that PET or PET/CT scanning may be useful in patients with an indeterminate fine-needle aspiration of thyroid nodules. ${ }^{37,38}$ In this setting, the test has a high negative predictive value, and has been proposed to be a way to reduce the number of hemithyroidectomies for what is ultimately proven to be a benign nodule. Another potential role for PET/CT in thyroid carcinoma is when recurrence is suspected (either clinically or due to elevated thyroglobulin) and radioiodine whole-body scintigraphy (WBS) is negative..$^{39-41}$ This situation is difficult from both a diagnostic and therapeutic standpoint. Since the tumor is not radioiodine avid it cannot be treated by radioiodine ablation. It also cannot be accurately localized by whole body scintigraphy to allow for surgical excision. In this 
scenario, the use of PET/CT has been shown to have a high positive predictive value and led to a change in management in greater than $40 \%$ of patients in 3 different studies. ${ }^{39-41}$ As PET imaging gains greater use in other diseases, the prevalence of PET incidentalomas of the thyroid will increase. In a review of 4,136 patients who underwent PET imaging for known non-thyroid malignancies, 45 demonstrated focal uptake with the thyroid gland. ${ }^{42}$ In $50 \%$ of the 32 patients for whom a cytologic diagnosis was available, the tumor was malignant. Given this high prevalence of malignancy, all PET incidentalomas of the thyroid must be evaluated for thyroid carcinoma.

2006 ATA Guidelines: Preoperative neck ultrasound for the contralateral lobe and cervical (central and bilateral) lymph nodes is recommended for all patients undergoing thyroidectomy for malignant cytologic findings on biopsy. Routine preoperative use of other imaging studies (CT, MRI, PET) is not recommended.

\section{Operative}

\section{Central Compartment}

Metastases from PTC occur frequently in the central compartment. The central compartment is generally defined as the space bounded by the common carotid arteries laterally, the hyoid bone superiorly, and the innominate vessels inferiorly. The recommendation for or against the routine dissection of the central compartment is based upon the risk of lymph node recurrence versus the morbidity of the procedure.

Some authors have suggested that due to the high frequency of central compartment metastases, that elective dissection of the central compartment should be performed in all patients with PTC. ${ }^{12,13,43}$ Others have attempted to actually prove that 
careful dissection of the central compartment at the time of thyroidectomy can positively influence patient survival. ${ }^{44}$ Ito et $\mathrm{al}^{9}$ reviewed 759 patients and demonstrated that the frequency of central node metastasis increased in relation to tumor size. They also showed that central node metastasis was an independent prognostic factor of disease-free survival in patients with PTC larger than $1 \mathrm{~cm}$. An argument against the routine performance of a CND in papillary thyroid carcinoma is that it has been shown to be associated with increased morbidity. Both Henry et $\mathrm{al}^{45}$ and Cheah et $\mathrm{al}^{46}$ showed that CND was associated with a statistically significant increased risk of both transient and permanent hypocalcemia. Roh et $\mathrm{al}^{7}$ were able to demonstrate that serum parathyroid hormone levels significantly decreased immediately after neck dissection in patients with PTC and remained low for several weeks when transient. In this study, the risk of hypocalcemia was further increased when LND was performed in addition to CND. None of these studies showed a statistically increased rate of recurrent laryngeal nerve injury with CND. Another argument for routine CND is that reoperation for recurrence is associated with significantly higher morbidity than an initial operation, and anything that can be done to minimize the risk of recurrence and reoperation should be employed. ${ }^{43}$ A 2007 evidence based review of the literature performed by White et $\mathrm{al}^{47}$ at the University of Michigan attempted to settle the debate on routine central compartment lymph node dissection. Their goal was to answer these 3 important questions: 1) Does central node dissection decrease recurrence or disease-specific mortality in PTC? 2) Does central node dissection increase the risk of hypothyroidism and recurrent laryngeal nerve injury? 3) Does reoperation in the central neck compartment for recurrent PTC increase the risk of hypoparathyroidism and recurrent laryngeal nerve injury? 
No prospective randomized data exist to answers these questions. After review of 13 studies, which include one prospective study and multiple retrospective cohort studies and case series, White et $\mathrm{al}^{47}$ concluded that based on the currently available evidence that CND "may" decrease recurrence and "likely" improves disease-specific survival. They also found that although CND may be associated with an increased risk of hypoparathyroidism and unintentional nerve injury at initial operation, the increased risk of these complications associated with reoperation for recurrence in the central neck compartment supports the more aggressive initial opertation. They concluded that evidence-based recommendations support CND for PTC in the hands of experienced surgeons.

The 2006 ATA guidelines ${ }^{5}$ are in accordance with the University of Michigan literature review. They recommend that routine central-compartment neck dissection should be considered for patients with PTC and suspected Hurthle cell carcinoma. Due to the low rate of metastases in FTC, they suggest that near-total or total thyroidectomy without CND may be appropriate for that diagnosis. Finally, they offer thyroidectomy without CND and followed by radioactive iodine (RAI) therapy as alternative approach for papillary and Hurthle cell cancers.

2006 ATA Guidelines: Routine central-compartment (level VI) neck dissection should be considered for patients with papillary thyroid carcinoma and suspected Hurthle carcinoma. Near-total or total thyroidectomy without central node dissection may be appropriate for follicular cancer, and when followed by radioactive iodine therapy, may provide an alternative approach for papillary and Hurthle cell cancers. 


\section{Lateral compartments}

In PTC, lymph node metastases are also common in the lateral neck, specifically the ipsilateral side. Since these metastases are frequently occult, discussion of the management of the lateral neck must be framed in the context of clinically negative and clinically positive.

The approach to the clinically negative neck has traditionally differed in Japan as compared to the United States. Studies from Japan indicate that patients were more likely to undergo a lateral neck dissection as part of the elective management for DTC., $22,33,48$ A contributing factor to this is that postoperative RAI therapy is rarely employed in Japan. Because of this, Japanese centers have provided insight into the effect of an elective neck dissection for DTC in the absence of RAI, specifically for PTMC (tumor size $<1 \mathrm{~cm}$ ). Wada et al $^{8}$ compared 235 patients who underwent prophylactic neck dissection for PTMC to 155 patients who underwent thyroidectomy for presumably benign disease and were discovered to have PTMC post-operatively. These 155 patients, therefore, did not undergo a neck dissection as part of there management and served as controls. Both groups were similar in regards to age, gender, and length of follow-up (greater than 53 months mean for both). They discovered that the recurrence rate did not differ between the prophylactic group and the "observation" group (0.43\% vs. $0.65 \%)$ and that it was low for both groups. They concluded that prophylactic neck dissection is not beneficial in patients with PTMC. Ito et $\mathrm{al}^{33}$ investigated 523 patients with PTMC who did not have detectable lateral node metastasis on ultrasonography. Of these patients, 249 underwent prophylactic LND and 274 did not. After a mean follow-up of over 4 years, there was no statistical difference in regards to lymph node recurrence-free survival. They also 
concluded that LND is not necessary in patients with PTMC without lymph node metastases detected on ultrasonography preoperatively.

Indeed, PTMC has a benign course. It is frequently found incidentally at autopsy and its discovery is a situation where completion thyroidectomy may be unnecessary according to the ATA guidelines. ${ }^{5,49}$ The prevalence of ultrasound-guided FNA biopsy has led to an increase in its diagnosis, thus forcing further consideration of its clinical significance. In another study, Ito et $\mathrm{al}^{34}$ offered a trial of observation for patients with PTMC. Of 732 patients, 162 opted for observation. During follow up, more than $70 \%$ of the primary tumors either did not change or decreased in size, and lateral compartment metastasis appeared in only $1.2 \%$ of patients. This is despite the fact that metastases were histologically confirmed in $50 \%$ of the patients in the surgically treated group. This again questions the clinical significance of occult nodal metastasis in PTMC. One limitation of the study is that the mean follow-up time was only approximately 4 years, which perhaps does not allow enough time for subclinical metastasis to become overt.

When PTC is larger than $1 \mathrm{~cm}$, the risk of nodal metastases is higher. ${ }^{9}$ Although there is arguably no effect on survival with nodal metastasis, there is the concern of an increased risk of recurrence. As was the case in the central compartment, some authors use the presence of the lymph nodes alone as an argument for a comprehensive approach to the lateral neck. ${ }^{10,15}$ The recommendations are made with the goal of reducing recurrence, but these studies have not yet completed the follow-up to prove that an aggressive approach with ipsilateral modified radical neck dissection (MRND) actually results in lower recurrences. No prospective, randomized trials exist to identify the best management of the lateral neck in thyroid cancer because of the limitations discussed 
earlier. ${ }^{50}$ And, unfortunately, the largest retrospective studies examining prognostic factors in DTC do not provide adequate detail to draw conclusions about the management of the neck. ${ }^{3,4,6,20,24}$

The NCDB report of 53,856 case of thyroid cancer treated in the US between 1985 and 1995 was able to observe that the majority of patients with DTC did not undergo lymph node dissection and that lymph node sampling was more popular than formal, comprehensive lymph node dissection. ${ }^{3}$ For PTC, for example, $14.6 \%$ of all patients underwent a limited lymph node dissection as part of their treatment, whereas $7.2 \%$ of patients had a radical or modified radical neck dissection. Another observation was that 38.3\% of patients with PTC within this population received radiotherapy (usually RAI) after surgery. The reported 10-year overall relative survival for PTC in this study was 93\%. However, without a means of identifying the indications for the above treatments and analysis of who received which combinations of surgery and radioactive iodine, there is no way to make meaningful conclusions on how the treatments affected outcomes. The Surveillance, Epidemiology and End Results (SEER) program is another large database $\left(15,698\right.$ patients) that is similarly limited by lack of treatment based analysis and controls. ${ }^{4}$ When lateral lymph node metastases are clinically apparent, the need for surgical excision is more obvious. In the past, lateral lymph nodes have been simply excised via "node picking" procedures, but these procedures are seldom advocated today. The current recommendation of the ATA guidelines ${ }^{5}$ is for functional compartmental en-bloc dissection for clinically positive nodes. Although this approach seems to be more oncologically sound, there are limited data to prove that it truly improves outcomes. In fact, in a group of 859 patients treated for PTC at the Mayo Clinic between 1946 and 1970 
when "node picking" was the more popular approach for cervical nodes and postoperative iodine was only used in $3 \%$ of patients, the nodal recurrence rate was only $6 \%{ }^{20}$ The overall mortality observed at 30 years was only $3 \%$ above that expected, with $6.5 \%$ of the patients dying from PTC. Unfortunately, this study failed to compare the effect of node picking (245/400) versus MRND (125/400) in regards to recurrence or mortality in their patients with cervical node excisions.

On the other hand, while only $6.5 \%$ of patients dying from the disease may look good in comparison to other types of cancer, a more aggressive approach that does not significantly increase morbidity would be warranted to further minimize mortality in this typically curable disease. In two studies looking at small groups of patients with DTC, Kupferman et al ${ }^{14,51}$ indicated that the pattern of lymph node metastases in patients with clinically positive necks warranted a comprehensive neck dissection with inclusion of levels II through V and that this procedure can be performed with acceptable morbidity.

In 2004, Wang at el ${ }^{52}$ reported a series of patients with DTC who were managed using the above suggested approach: LND only for clinically palpable nodes, and MRND with preservation of the submandibular triangle when these nodes were excised. Of the 508 patients, 44 required a neck dissection for clinically palpable lateral nodes. Only $3 \%$ of the 464 patients who did not undergo initial neck dissection recurred in the lateral cervical nodes. They discovered that recurrence was more likely when the initial tumor is larger than $4 \mathrm{~cm}$. Of the 16 patients who recurred in the lateral neck, 5 were younger than 45 years and were alive and free of disease. Of the 11 who recurred and were over 45 years, however, 4 had died of their thyroid cancer. This led them to conclude that aggressive attempts to detect and treat occult lateral cervical nodes was for the most part 
unnecessary, but could be considered in older patients with large primary tumors. Lebouleux et $\mathrm{al}^{23}$ examined 148 patients with lymph node metastases (140 patients) or extrathyroidal extension (8 patients) who underwent total thyroidectomy with central and ipsilateral en bloc neck dissection of levels III and IV followed by RAI. The neck dissection was extended to include levels II and V if frozen section of the levels III and IV showed metastatic involvement. Of these high-risk patients, $22 \%$ had persistent disease on the post-ablation WBS. This allowed them to identify significant risk factors for persistent disease, which include $>10$ lymph node metastases, lymph nodes with extracapsular extension, and tumor size $>4 \mathrm{~cm}$. Still, the ten-year disease specific survival was 99\%, and the recurrence rate for the patients with a normal post-ablation WBS was $7 \%$ with an 8 year mean follow up.

The ATA guidelines agree with the findings and recommendations of these two studies. ${ }^{5}$ They suggest that a compartmental lymph node dissection should be performed for patients with biopsy-proven metastatic cervical lymphadenopathy, especially when they are likely to fail radioactive iodine.

Dissection of the lateral compartment can be accomplished through a single transverse incision by extending the thyroidectomy incision laterally to the anterior border of the trapezius. A second transverse incision (MacFee incision) may be added superiorly, when necessary, to facilitate exposure of the upper neck nodes. Alternatively, a modified apron incision that incorporates the thyroidectomy incision can be used.

\section{ATA Guidelines: Lateral neck compartmental lymph node dissection should be}

performed for patients with biopsy-proven metastatic cervical lymphadenopathy detected

clinically or by imaging, especially when they are likely to fail radioactive iodine 
treatment based on lymph node size, number, or other factors, such as aggressive

\section{histology of the primary tumor.}

\section{Sentinel Node Biopsy}

Sentinel lymph node (SLN) biopsy is a technique that has gained wide acceptance in the management of breast cancer and melanoma. Recently, it has been offered as an option in the management of DTC. Fukui et al ${ }^{53}$ performed SLN biopsy in 22 patients by injecting methylene blue around the primary tumor site at the time of surgery. They proceeded to perform MRND in all of the patients. They reported a $90.5 \%$ concordance rate between the SLN findings and the regional lymph node status. Dzodic ${ }^{54}$ offered the SLN biopsy as means to determine which patients with clinically negative necks could benefit from MRND. He performed the procedure in 40 patients with DTC and had a 92.5\% SLN identification rate. Although the procedure can be done, it is unclear if it would be beneficial in DTC. Breast cancer and melanoma differ from DTC in that lymph node metastasis has a definite and important impact on prognosis for these tumor types. Additionally, regional lymphadenectomy for these cancers is associated with significant morbidity. Currently, neck dissection is only recommended for clinically or ultrasound apparent nodal metastases in patients with DTC. ${ }^{5}$ Because the impact of occult lymph node metastasis on survival in DTC has been shown to be low and possibly insignificant, it is unclear whether SLN biopsy would alter outcomes. The influence of occult lymph node metastasis on recurrence is likely higher, however, and if occult cervical metastases could in any way account for the small percentage of patients who die of DTC, then their identification would be beneficial. The ATA guidelines ${ }^{5}$ suggest that future research must 
be aimed at developing techniques to identify small cervical metastases in DTC for these reasons. SLN biopsy has potential to fulfill that goal. A large study demonstrating not only its feasibility and accuracy, but more importantly its benefit, must be performed before SLN is adopted in the workup of DTC.

\section{Minimally invasive/endoscopic neck dissection}

SLN biopsy was developed as a method to minimize morbidity by preventing unnecessary en bloc lymph node dissections. Minimally invasive and endoscopic techniques are proposed as methods to minimize morbidity when lymph node dissections are necessary. Additionally, a minimally invasive approach to thyroidectomy is gaining in popularity, which will likely lead to increased patient awareness and demand for the technique within this patient population. Terris et a ${ }^{55}$ demonstrated the feasibility of an endoscopic selective neck dissection using a porcine model. Lombardi et $\mathrm{al}{ }^{56}$ recently described a minimally invasive technique using video assistance for performing a lateral neck dissection, which they employed in 2 patients with PTC. Additionally, the combination of SLN biopsy with an endoscopic approach has been offered as an initial low-morbidity staging procedure. ${ }^{57}$ This would allow for an easy conversion to an endoscopic neck dissection if the sentinel node proves to be positive. As with SLN biopsy, however, the role of this technique in DTC is limited due to the apparent lack of significance of occult lymph node metastasis. Therefore, minimally invasive techniques must be proven to be safe and effective for $\mathrm{N}+$ necks, or prophylactic dissection of the lateral neck in DTC must be proven to be beneficial before endoscopic-assisted neck dissection can be considered useful in the management of DTC. Indeed, if occult 
metastases are proven to affect prognosis, then SLN biopsy and minimally invasive techniques could play an important role in the future management of the neck in DTC.

\section{Conclusions}

DTC is a relatively rare malignancy carrying a favorable prognosis. Survival rates exceed $90 \%$, but patients often survive with evidence of distant disease and die of unrelated causes, pointing to a relatively indolent but potentially lethal process. Despite a high long-term survival rate, recurrences are common and death from DTC does occur, even if in less than $10 \%$ of patients. An appropriately aggressive treatment of DTC must limit the risk of recurrence and death while minimizing treatment morbidity and recognizing the indolent course that DTC follows in most patients. This is especially true of the management of the neck, where lymph node metastases occur frequently and are associated with an unclear effect on prognosis. The 2006 updated treatment guidelines by the ATA are based on the currently available data, and make an effort to reach that treatment balance. An aggressive compartment oriented neck dissection is recommended for patients with evidence of cervical lymph node metastasis on physical exam or ultrasonograpy, but elective dissection of the negative lateral neck is not.

The ATA guidelines are based largely on retrospective reviews because the infrequency of the disease combined with its high survival rate limits the practicality of a large randomized prospective trial. Although a randomized prospective trial would provide the best evidence for management protocols, the ATA guidelines are a valuable interpretation of the current evidence, which at times is conflicting. A uniform approach to the management of DTC should limit the chance of undertreatment and should improve 
the ability to analyze outcomes in the future. Both would aid in further lowering the risk of recurrence and death from thyroid cancer.

\section{REFERENCES}

1. American Cancer Society. Cancer Facts and Figures 2007. Atlanta: American Cancer Society; 2007.

2. Shaha AR. Implications of prognostic factors and risk groups in the management of differentiated thyroid cancer. Laryngoscope. 2004;114:393-402.

3. Hundahl SA, Fleming ID, Fremgen AM, Menck HR. A national cancer data base report on 53,856 cases of thyroid carcinoma treated in the U.S., 1985-1995. Cancer. 1998;83:2638-2648.

4. Gilliland FD, Hunt WC, Morris DM, Key CR. Prognostic factors for thyroid carcinoma: A population-based study of 15,698 cases from the surveillance, epidemiology and end results (SEER) program 1973-1991. Cancer. 1997;79:564-573.

5. Cooper DS, Doherty GM, Haugen BR, et al. Management guidelines for patients with thyroid nodules and differentiated thyroid cancer. Thyroid. 2006;16:109-141.

6. Shaha AR, Shah JP, Loree TR. Patterns of nodal and distant metastasis based on histologic varieties in differentiated carcinoma of the thyroid. Am J Surg. 1996;172:692-694. 
7. Roh J-, Park J-, Park CI. Total thyroidectomy plus neck dissection in differentiated papillary thyroid carcinoma patients: Pattern of nodal metastasis, morbidity, recurrence, and postoperative levels of serum parathyroid hormone. Annals of Surgery. 2007;245:604-610.

8. Wada N, Duh Q-, Sugino K, et al. Lymph node metastasis from 259 papillary thyroid microcarcinomas: Frequency, pattern of occurrence and recurrence, and optimal strategy for neck dissection. Annals of Surgery. 2003;237:399-407.

9. Ito Y, Jikuzono T, Higashiyama T, et al. Clinical significance of lymph node metastasis of thyroid papillary carcinoma located in one lobe. World Journal of Surgery. 2006;30:1821-1828.

10. Goropoulos A, Karamoshos K, Christodoulou A, et al. Value of the cervical compartments in the surgical treatment of papillary thyroid carcinoma. World Journal of Surgery. 2004;28:12751281.

11. Ito Y, Tomoda C, Uruno T, et al. Clinical significance of metastasis to the central compartment from papillary microcarcinoma of the thyroid. World Journal of Surgery. 2006;30:91-99.

12. Qubain SW, Nakano S, Baba M, Takao S, Aikou T. Distribution of lymph node micrometastasis in pN0 well-differentiated thyroid carcinoma. Surgery. 2002;131:249-256.

13. Gimm O, Rath FW, Dralle H. Pattern of lymph node metastases in papillary thyroid carcinoma. British Journal of Surgery. 1998;85:252-254.

14. Kupferman ME, Patterson M, Mandel SJ, LiVolsi V, Weber RS. Patterns of lateral neck metastasis in papillary thyroid carcinoma. Archives of Otolaryngology - Head and Neck Surgery. 2004;130:857-860.

15. Machens A, Hinze R, Thomusch O, Dralle H. Pattern of nodal metastasis for primary and reoperative thyroid cancer. World Journal of Surgery. 2002;26:22-28. 
16. Sivanandan R, Soo KC. Pattern of cervical lymph node metastases from papillary carcinoma of the thyroid. British Journal of Surgery. 2001;88:1241-1244.

17. Scheumann GFW, Gimm O, Wegener G, Hundeshagen H, Dralle H, Cady B. Prognostic significance and surgical management of locoregional lymph node metastases in papillary thyroid cancer. World Journal of Surgery. 1994;18:559-568.

18. Shaha AR, Shah JP, Loree TR. Risk group stratification and prognostic factors in papillary carcinoma of thyroid. Annals of Surgical Oncology. 1996;3:534-538.

19. Hughes CJ, Shaha AR, Shah JP, Loree TR. Impact of lymph node metastasis in differentiated carcinoma of the thyroid: A matched-pair analysis. Head and Neck. 1996;18:127-132.

20. McConahey WM, Hay ID, Woolner LB, van Heerden JA, Taylor WF. Papillary thyroid cancer treated at the mayo clinic, 1946 through 1970: Initial manifestations, pathologic findings, therapy, and outcome. Mayo Clin Proc. 1986;61:978-996.

21. Mazzaferri EL, Jhiang SM. Long-term impact of initial surgical and medical therapy on papillary and follicular thyroid cancer. Am J Med. 1994;97:418-428.

22. Moley JF, Wells SA. Compartment-mediated dissection for papillary thyroid cancer. Langenbeck's Archives of Surgery. 1999;384:9-15.

23. Leboulleux S, Rubino C, Baudin E, et al. Prognostic factors for persistent or recurrent disease of papillary thyroid carcinoma with neck lymph node metastases and/or tumor extension beyond the thyroid capsule at initial diagnosis. Journal of Clinical Endocrinology and Metabolism. 2005;90:5723-5729.

24. Shaha AR, Shah JP, Loree TR. Low-risk differentiated thyroid cancer: The need for selective treatment. Annals of Surgical Oncology. 1997;4:328-333. 
25. Scheumann GF, Gimm O, Wegener G, Hundeshagen H, Dralle H. Prognostic significance and surgical management of locoregional lymph node metastases in papillary thyroid cancer. World $J$ Surg. 1994;18:559-67; discussion 567-8.

26. Lundgren CI, Hall P, Dickman PW, Zedenius J. Clinically significant prognostic factors for differentiated thyroid carcinoma: A populationed-based, nested case-control study. Cancer. 2006;106:524-531.

27. Sellers M, Beenken S, Blankenship A, et al. Prognostic significance of cervical lymph node metastases in differentiated thyroid cancer. Am J Surg. 1992;164:578-581.

28. Kouvaraki MA, Shapiro SE, Fornage BD, et al. Role of preoperative ultrasonography in the surgical management of patients with thyroid cancer. Surgery. 2003;134:946-955.

29. Sanders LE, Rossi RL. Occult well differentiated thyroid carcinoma presenting as cervical node disease. World J Surg. 1995;19:642-646.

30. Frasoldati A, Toschi E, Zini M, et al. Role of thyroglobulin measurement in fine-needle aspiration biopsies of cervical lymph nodes in patients with differentiated thyroid cancer. Thyroid. 1999;9:105-111.

31. Cunha N, Rodrigues F, Curado F, et al. Thyroglobulin detection in fine-needle aspirates of cervical lymph nodes: A technique for the diagnosis of metastatic differentiated thyroid cancer. Eur J Endocrinol. 2007;157:101-107.

32. Cignarelli M, Ambrosi A, Marino A, et al. Diagnostic utility of thyroglobulin detection in fineneedle aspiration of cervical cystic metastatic lymph nodes from papillary thyroid cancer with negative cytology. Thyroid. 2003;13:1163-1167. 
33. Ito Y, Tomoda C, Uruno T, et al. Preoperative ultrasonographic examination for lymph node metastasis: Usefulness when designing lymph node dissection for papillary microcarcinoma of the thyroid. World Journal of Surgery. 2004;28:498-501.

34. Ito Y, Uruno T, Nakano K, et al. An observation trial without surgical treatment in patients with papillary microcarcinoma of the thyroid. Thyroid. 2003;13:381-387.

35. Shimamoto K, Satake H, Sawaki A, Ishigaki T, Funahashi H, Imai T. Preoperative staging of thyroid papillary carcinoma with ultrasonography. Eur J Radiol. 1998;29:4-10.

36. Hoang JK, Lee WK, Lee M, Johnson D, Farrell S. US features of thyroid malignancy: Pearls and pitfalls. Radiographics. 2007;27:847-860.

37. De Geus-Oei L-, Pieters GFFM, Bonenkamp JJ, et al. 18F-FDG PET reduces unnecessary hemithyroidectomies for thyroid nodules with inconclusive cytologic results. Journal of Nuclear Medicine. 2006;47:770-775.

38. Mitchell JC, Grant F, Evenson AR, et al. Preoperative evaluation of thyroid nodules with 18FDG-PET/CT. Surgery. 2005;138:1166-1175.

39. Palmedo H, Bucerius J, Joe A, et al. Integrated PET/CT in differentiated thyroid cancer: Diagnostic accuracy and impact on patient management. Journal of Nuclear Medicine. 2006;47:616-624.

40. Nahas Z, Goldenberg D, Fakhry C, et al. The role of positron emission tomography/computed tomography in the management of recurrent papillary thyroid carcinoma. Laryngoscope. 2005;115:237-243. 
41. Shammas A, Degirmenci B, Mountz JM, et al. 18F-FDG PET/CT in patients with suspected recurrent or metastatic well-differentiated thyroid cancer. Journal of nuclear medicine : official publication, Society of Nuclear Medicine. 2007;48:221-226.

42. Kim TY, Kim WB, Ryu JS, Gong G, Hong SJ, Shong YK. 18F-fluorodeoxyglucose uptake in thyroid from positron emission tomogram (PET) for evaluation in cancer patients: High prevalence of malignancy in thyroid PET incidentaloma. Laryngoscope. 2005;115:1074-1078.

43. Shindo M, Wu JC, Park EE, Tanzella F. The importance of central compartment elective lymph node excision in the staging and treatment of papillary thyroid cancer. Archives of Otolaryngology - Head and Neck Surgery. 2006;132:650-654.

44. Tisell L-, Nilsson B, Molne J, et al. Improved survival of patients with papillary thyroid cancer after surgical microdissection. World Journal of Surgery. 1996;20:854-859.

45. Henry JF, Gramatica L, Denizot A, Kvachenyuk A, Puccini M, Defechereux T. Morbidity of prophylactic lymph node dissection in the central neck area in patients with papillary thyroid carcinoma. Langenbeck's Archives of Surgery. 1998;383:167-169.

46. Cheah WK, Arici C, Ituarte PHG, Siperstein AE, Duh Q-, Clark OH. Complications of neck dissection for thyroid cancer. World Journal of Surgery. 2002;26:1013-1016.

47. White ML, Gauger PG, Doherty GM. Central lymph node dissection in differentiated thyroid cancer. World J Surg. 2007;31:895-904.

48. Noguchi M, Kumaki T, Taniya T, et al. Impact of neck dissection on survival in welldifferentiated thyroid cancer: A multivariate analysis of 218 cases. Int Surg. 1990;75:220-224.

49. Yamamoto Y, Maeda T, Izumi K, Otsuka H. Occult papillary carcinoma of the thyroid. A study of 408 autopsy cases. Cancer. 1990;65:1173-1179. 
50. Shaha AR. TNM classification of thyroid carcinoma. World Journal of Surgery. 2007;31:879887.

51. Kupferman ME, Patterson DM, Mandel SJ, LiVolsi V, Weber RS. Safety of modified radical neck dissection for differentiated thyroid carcinoma. Laryngoscope. 2004;114:403-406.

52. Wang TS, Dubner S, Sznyter LA, Heller KS. Incidence of metastatic well-differentiated thyroid cancer in cervical lymph nodes. Archives of Otolaryngology - Head and Neck Surgery. 2004;130:110-113.

53. Fukui Y, Yamakawa T, Taniki T, Numoto S, Miki H, Monden Y. Sentinel lymph node biopsy in patients with papillary thyroid carcinoma. Cancer. 2001;92:2868-2874.

54. Dzodic R. Sentinel lymph node biopsy may be used to support the decision to perform modified radical neck dissection in differentiated thyroid carcinoma. World Journal of Surgery. 2006;30:841-846.

55. Terris DJ, Monfared A, Thomas A, Kambham N, Saenz Y. Endoscopic selective neck dissection in a porcine model. Arch Otolaryngol Head Neck Surg. 2003;129:613-617.

56. Lombardi CP, Raffaelli M, Princi P, De Crea C, Bellantone R. Minimally invasive videoassisted functional lateral neck dissection for metastatic papillary thyroid carcinoma. Am J Surg. 2007;193:114-118.

57. Malloy KM, Cognetti DM, Wildemore BM, et al. Feasibility of endoscopic sentinel node biopsy in the porcine neck. Otolaryngol Head Neck Surg. 2007;136:806-810. 